\title{
RESEARCH
}

Open Access

\section{Health-related quality of life and its risk factors in Chinese hereditary angioedema patients}

\author{
Shuang Liu ${ }^{1,2}$, Xue Wang ${ }^{1,3}$, Yingyang $X u^{1}$, Qun $X u^{4,5}$ and Yuxiang Zhi ${ }^{{ }^{*}}$ (D)
}

\begin{abstract}
Background: Hereditary angioedema (HAE) is a rare but serious condition characterized by unpredictable and recurrent attacks affecting the skin and mucosa. HAE has wide-ranging impacts on the health-related quality of life (HRQoL) of patients. This study aims to assess the HRQoL of Chinese patients with HAE using the 36-item Short Form Health Survey (SF-36v2) and to explore potential risk factors for low HRQoL.

Methods: A total of 104 patients (47 male and 57 female) over age 18 living in China with a known diagnosis of HAE due to C1-INH deficiency completed the SF-36V2 (generic HRQoL questionnaire). The results were compared to Chinese population norms. Subgroup analysis and logistic regression were used to interpret the data.

Results: SF-36V2 showed a significant reduction in all dimensions of HRQOL $(p<0.001)$ in patients with HAE compared with the general Chinese population. Female patients reported significantly lower bodily pain (BP) ( $p=$ $0.039)$ and physical component scores (PCSs) $(p=0.027)$ than male patients. Patients with mucosal edema tended to report lower role-physical (RP) limitations $(p=0.031)$ than patients with only skin edema. There were no differences between the mean scores of the SF-36 in relation to disease subtype, age, disease severity and longterm prophylaxis. Among female patients on long-term prophylaxis, social functioning (SF) $(r=-0.404, p=0.010)$, role-emotional (RE) $(r=-0.320, p=0.044)$ and mental component scores (MCSs) $(r=-0.313, p=0.049)$ were negatively correlated with danazol dosage. A correlation between decreased disease control and decreased HRQoL scores was found, although the correlation was not significant in terms of RE or mental health (MH) scores. The logistic regression model revealed uncontrolled disease to be a risk factor for a low PCS (odds ratio 10.77, 95\% confidence interval $[\mathrm{Cl}]$ 1.78-65.06; $p=0.010$ ) and laryngeal edema to be a risk factor for a low MCS (odds ratio 4.75, 95\% Cl 1.09-20.69; $p=0.038)$.
\end{abstract}

Conclusions: Chinese HAE patients reported significantly lower HRQoL scores than the general population. Unsatisfactory disease control is a risk factor for decreased PCSs. Laryngeal edema is a risk factor for decreased MCSs.

Keywords: Hereditary angioedema, Health-related quality of life, Chinese, SF-36

\footnotetext{
*Correspondence: yuxiang_zhi@126.com

'Department of Allergy, Peking Union Medical College Hospital, Chinese Academy of Medical Sciences and Peking Union Medical College, Beijing 100730, China

Full list of author information is available at the end of the article
}

(c) The Author(s). 2019 Open Access This article is distributed under the terms of the Creative Commons Attribution 4.0 International License (http://creativecommons.org/licenses/by/4.0/), which permits unrestricted use, distribution, and reproduction in any medium, provided you give appropriate credit to the original author(s) and the source, provide a link to the Creative Commons license, and indicate if changes were made. The Creative Commons Public Domain Dedication waiver (http://creativecommons.org/publicdomain/zero/1.0/) applies to the data made available in this article, unless otherwise stated. 


\section{Introduction}

Hereditary angioedema (HAE), a rare autosomal dominant disorder, is characterized by unpredictable and recurrent attacks of painful swelling that typically affect the extremities, bowel mucosa, genitals, face and upper airways [1]. Several forms of HAE have been defined: [1] type 1 HAE (HAE-1), which is caused by $\mathrm{C} 1$ inhibitor (C1-INH) deficiency and characterized by low C1-INH level and function; [2] type $2 \mathrm{HAE}$ (HAE-2), which results from $\mathrm{C} 1-\mathrm{INH}$ dysfunction and is characterized by normal or slightly higher $\mathrm{C} 1-\mathrm{INH}$ levels but impaired function; and [3] HAE with normal C1 inhibitor level and function, which is caused by a mutation in the F12 gene (HAE-FXII), the angiopoietin-1 gene (HAEANGPT1), in the plasminogen gene (HAE-PLG) and in unknown genes (HAE-UNK) [2]. The pathogenesis remains unknown in some HAE patients. Inherited in an autosomal dominant manner, HAE due to C1-INH (C1-INH HAE) has an estimated prevalence of 1.1$1.6 / 100,000$ with no gender difference [3]. The variability in disease manifestations and difficulties in differentiating HAE symptoms from those of more common angioedema subtypes account for a tendency of misdiagnosis and lead to a diagnostic delay typically longer than 10 years [4]. In addition, a considerable proportion of patients experience potentially lethal laryngeal edema as the disease progresses [5]. Therefore, HAE poses a serious threat to a patient's life expectancy and quality of life.

Health-related quality of life (HRQoL) is defined as the subjective perception by patients of the multidimensional impacts of a disease or condition [6]. HRQoL assessments could provide patients, healthcare providers and decision makers with more comprehensive information concerning patient health status, disease burden and therapeutic response. A series of validated questionnaires has been used to measure the HRQoL of HAE patients, including generic assessments (e.g., the Short Form 36 Health Survey [SF-36] [7]) and specific questionnaires for a certain disease or condition (e.g., the Angioedema Quality of Life Questionnaire [AE-QoL] [8]). Generic tools make it possible to compare the HRQoL between different diseases, while specific questionnaires are better at reflecting certain characteristics of a particular disease or condition.

HAE produces wide-ranging impacts in patients across physical health $(\mathrm{PH})$ and mental health $(\mathrm{MH})$ domains. Its unpredictable edematous attacks can cause disfigurement and severe bodily pain (BP), thereby negatively impacting the educational attainment, career advancement and social activity of patients [9]. Laryngeal edema can lead to upper airway obstruction, asphyxiation and even death [10]. Gastrointestinal attacks, which cause severe pain, are often mistaken for acute abdomen, resulting in unnecessary surgical interventions [11]. In addition, increased levels of depression and anxiety have been reported by several studies [12, 13]. Bonner et al. proposed a patient-reported outcome (PRO) in which the impacts of HAE attacks on different aspects of life were elaborated [14].

The first HRQoL study for patients with HAE was published in 1999; in this study, the Dermatology Life Quality Index (DLQI) was explored to measure the disability in different urticarial groups, including C1-INHHAE patients [15]. Short Form Health Surveys (including SF-12 and SF-36) are the most widely applied tools in generic HRQoL assessments. Lumry et al. used SF-12 to evaluate HRQoL in US C1-INH-HAE patients and found impaired $\mathrm{PH}$ and $\mathrm{MH}$ in comparison to those of the general population [9]. Studies in France [16], Sweden [17], Denmark [18], Brazil [19], Canada [7], the United Kingdom [20] and Colombia [21] have reached similar conclusions. In addition, increasingly more therapeutic studies have incorporated HRQoL assessments in the outcome measures in recent years [22-24]. Caballero et al. summarized the main research findings concerning HRQoL studies on HAE patients in 2017 [6].

Despite the emergence of numerous treatment advances, until now, no acute attack medication has been approved in China. The management is limited to fresh frozen plasma (FFP) transfusion and long-term prophylaxis with danazol or tranexamic acid. Therefore, studies in other countries do not necessarily represent the current state of the HRQoL in China. The HRQoL status in Chinese HAE patients has not yet been satisfactorily described. The aim of this study was to assess the HRQoL of Chinese patients with HAE using the 36-item Short Form Health Survey (SF-36v2) and to explore potential risk factors for decreased HRQoL.

\section{Results}

Demographic and clinical characteristics of the patients

One hundred and four patients completed this study. Baseline demographic and clinical characteristics are shown in Table 1.

\section{Scores of SF-36 in Chinese HAE patients}

Figure 1 outlines the SF-36 mean score for 8 specific dimensions. In all dimensions, HAE patients score significantly lower than the general population (Fig. 1). The physical component score (PCS) and mental component score (MCS) of participants are $49.81 \pm 7.08$ and $44.76 \pm$ 9.18, respectively. Scores of each dimension are shown in Additional file 1: Table S1.

Female patients reported lower scores than male patients in all eight dimensions and two summary component scores; however, only the differences in terms of BP $(p=0.039)$ and the PCS $(p=0.027)$ were significant. 
Table 1 Baseline characteristics of the 104 hereditary angioedema patients

\begin{tabular}{|c|c|}
\hline & $\mathrm{N}(\%)$ or median [IQR] \\
\hline Total & $104(100.0)$ \\
\hline \multicolumn{2}{|l|}{ C1-INH HAE diagnosis } \\
\hline Type 1 & $101(97.1)$ \\
\hline Type 2 & $3(2.9)$ \\
\hline \multicolumn{2}{|l|}{ Sex } \\
\hline Male & $47(45.2)$ \\
\hline Female & $57(54.8)$ \\
\hline \multicolumn{2}{|l|}{ Age (years) } \\
\hline $18-30$ & $27(26.0)$ \\
\hline $31-44$ & $52(50.0)$ \\
\hline$\geq 45$ & $25(24.0)$ \\
\hline \multicolumn{2}{|l|}{ Age at symptom onset (years) } \\
\hline $0-10$ & $16(15.4)$ \\
\hline $11-20$ & $54(51.9)$ \\
\hline$\geq 21$ & $34(32.7)$ \\
\hline Annual attack frequency $(n=75)$ & $3.0[0.8-6.0]$ \\
\hline \multicolumn{2}{|c|}{ Attacks in the 4 weeks before HRQoL measurement } \\
\hline Positive & $49(47.1)$ \\
\hline Negative & $55(52.9)$ \\
\hline \multicolumn{2}{|l|}{ Current skin edema } \\
\hline Positive & $81(77.9)$ \\
\hline Negative & $23(22.1)$ \\
\hline \multicolumn{2}{|l|}{ Current Gl edema } \\
\hline Positive & $58(55.8)$ \\
\hline Negative & $46(44.2)$ \\
\hline \multicolumn{2}{|l|}{ Current laryngeal edema } \\
\hline Positive & $26(25.0)$ \\
\hline Negative & $78(75.0)$ \\
\hline \multicolumn{2}{|l|}{ Severity evaluation } \\
\hline Mild & $39(37.5)$ \\
\hline Moderate & $20(19.2)$ \\
\hline Severe & $45(43.3)$ \\
\hline \multicolumn{2}{|l|}{ Disease control status } \\
\hline Completely controlled & 35 (33.6) \\
\hline Partly controlled & $55(52.9)$ \\
\hline Not controlled & $14(13.5)$ \\
\hline \multicolumn{2}{|l|}{ Long-term prophylaxis } \\
\hline Positive & $74(71.2)$ \\
\hline Negative & $30(28.8)$ \\
\hline \multicolumn{2}{|l|}{ Family history } \\
\hline Positive & $72(69.2)$ \\
\hline Negative & $32(30.8)$ \\
\hline
\end{tabular}

When we stratified the patients into patients with mucosal edema and those without, a significant difference was observed in terms of RP. Patients with mucosal edema tended to report lower RP $(p=0.031)$ than patients with only skin edema. Compared to patients with only laryngeal or gastrointestinal edema, those with both laryngeal and gastrointestinal edema did not show significant differences in any dimension or summary score.

No significant differences were observed between type 1 and 2 patients. When stratifying patients into three subgroups according to age, i.e., 18-30, 31-44, and over 45 , we found no significant differences in any dimension or component summary score. Severity classification was not found to be related to SF-36 scores.

No statistically significant correlation was found between the clinical severity score and SF-36 scores in terms of eight dimensions and two component summary scores (Additional file 2: Table S2).

\section{Relation between disease control and HRQoL}

Among the 104 patients in this study, 35 reported that their edematous attacks were completely controlled (i.e., they experienced no more attacks after disease management), 55 reported that their attacks were partly controlled (i.e., they suffered from fewer attacks after diagnosis and management such as taking long/short-term prophylaxis and avoiding triggers), and 14 reported no improvement of the disease after diagnosis and management (i.e., the disease was not controlled). A correlation between poor disease control and decreased HRQoL scores was found, although the correlation was not significant in terms of RE and $\mathrm{MH}$. The PCS was also significantly correlated with disease control status. Additional file 3: Table S3 shows the correlation between disease control and HRQoL.

In all dimensions except RE, significant differences were found between the three subgroups. Table 2 shows the HRQoL scores in three subgroups (Kruskal-Wallis rank-sum test).

Negative correlations between attacks in the 4 weeks before HRQoL measurement and all dimensions of HRQoL were found, although the correlations were not significant in terms of physical functioning (PF), VT and $\mathrm{MH}$. The PCS was also significantly correlated with attacks in the 4 weeks before HRQoL measurement

\section{Impacts of long-term prophylaxis on HRQoL}

In this study, 74 patients were under long-term prophylaxis with danazol. Fifteen patients discontinued danazol prophylaxis due to unbearable side effects or just worrying about side effects. Seventeen patients had never initiated danazol prophylaxis. However, no significant difference was found between patients under long-term prophylaxis and those not under long-term prophylaxis. No correlation was found between HRQoL and danazol 


\section{HAE patients vs. Chinese population norms \\ HAE patients \\ Chinese norm}

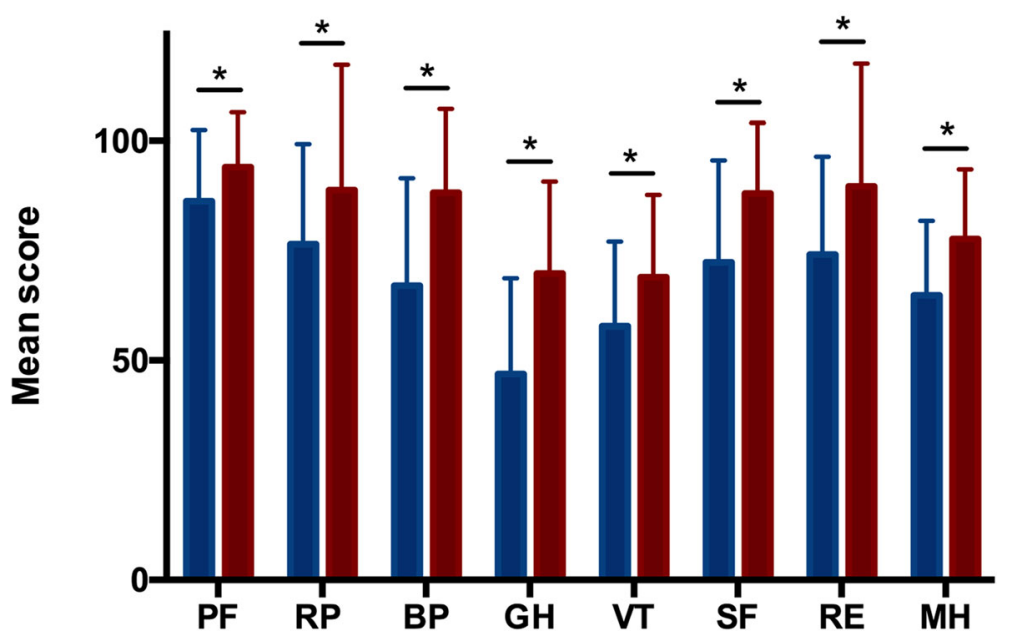

Fig. 1 Mean scores in SF-36v2 for Chinese hereditary angioedema (HAE) patients vs. Chinese population norms. PF physical functioning, RP rolephysical, BP bodily pain, GH general health, VT vitality, SF social functioning, RE role-emotional, $M H$ mental health. ${ }^{*} p<0.001$

dosage when all patients were analyzed on the whole. When the patients were stratified according to sex, the correlations between SF $(\mathrm{r}=-0.404, p=0.010), \mathrm{RE}(\mathrm{r}=$ $-0.320, p=0.044)$, MCS $(r=-0.313, p=0.049)$ and danazol dosage were significant in women.

\section{Risk factors for low health-related quality of life}

We stratified patients into two groups according to the PCS with a cutoff point of 50 and investigated the relationship between the PCS and potential risk factors. HAE patients with current GI edema, laryngeal edema and unsatisfactory disease control had significantly $(p<0.05)$ lower PCSs. A logistic regression model revealed that uncontrolled disease was a risk factor for low PCSs (Table 3).
We then stratified the patients into two groups according to the MCS with a cutoff point of 50 and investigated the relationship between the MCS and potential risk factors. HAE patients with current laryngeal edema and unideal disease control had significantly $(\mathrm{p}<0.05)$ lower MCSs. Logistic regression analysis indicated that current laryngeal edema (odds ratio 4.933, 95\% confidence interval [CI] 1.154-21.094; $\mathrm{p}=0.031$ ) was independently associated with an MCS $<50$ in HAE patients (Table 4.).

\section{Discussion}

This study is the first to perform a comprehensive analysis of HRQoL in Chinese HAE patients. Since no acute attack medication has been available on the market, the

Table 2 Median score in the 8 dimensions and 2 component summary scores of SF-36 grouped by disease control status

\begin{tabular}{lllll}
\hline Dimension & Completely controlled $(n=35)$ & Partly controlled $(n=55)$ & Not controlled $(n=14)$ & $P$ value (Kruskal-Wallis test) \\
\hline PF & $100.0[80.0-100.0]$ & $90.0[80.0-100.0]$ & $70.0[58.8-92.5]$ & 0.004 \\
RP & $100.0[75.0-100.0]$ & $75.0[62.5-93.8]$ & $75.0[48.4-84.4]$ & 0.010 \\
BP & $80.0[62.0-100.0]$ & $74.0[41.0-84.0]$ & $52.0[38.8-80.5]$ & 0.044 \\
GH & $52.0[35.0-72.0]$ & $47.0[32.0-60.0]$ & $30.0[10.0-42.8]$ & 0.003 \\
VT & $68.8[50.0-75.0]$ & $62.5[43.8-68.8]$ & $43.8[25.0-50.0]$ & 0.002 \\
SF & $87.5[62.5-100.0]$ & $75.0[62.5-87.5]$ & $62.5[37.5-75.0]$ & 0.016 \\
RE & $75.0[58.3-100.0]$ & $75.0[50.0-91.7]$ & $75.0[50.0-85.4]$ & 0.191 \\
MH & $70.0[55.0-80.0]$ & $70.0[60.0-75.0]$ & $57.5[35.0-65.0]$ & 0.005 \\
PCS & $53.5[49.0-58.1]$ & $50.0[45.8-54.4]$ & $43.2[38.6-52.3]$ & 0.002 \\
MCS & $45.7[40.9-51.9]$ & $47.4[39.8-51.6]$ & $40.9[34.7-45.5]$ & 0.028
\end{tabular}

The data are summarized as median plus interquartile range [IQR] 
Table 3 Logistic regression analysis of HAE patients with a PCS $<50$

\begin{tabular}{|c|c|c|}
\hline & \multicolumn{2}{|c|}{ Logistic regression results } \\
\hline & $\mathrm{OR}[95 \% \mathrm{Cl}]$ & $P$ value \\
\hline \multicolumn{3}{|l|}{ Sex } \\
\hline Female & $2.28[0.92,5.68]$ & 0.075 \\
\hline \multicolumn{3}{|l|}{ Age } \\
\hline $31-44$ & $0.46[0.15,1.47]$ & 0.190 \\
\hline$\geq 45$ & $0.54[0.130,1.817]$ & 0.374 \\
\hline \multicolumn{3}{|l|}{ Onset age } \\
\hline $11-20$ & $0.46[0.13,1.70]$ & 0.246 \\
\hline$\geq 21$ & $0.64[0.15,2.79]$ & 0.556 \\
\hline \multicolumn{3}{|l|}{ Current skin edema } \\
\hline Positive & $0.57[0.15,2.14]$ & 0.408 \\
\hline \multicolumn{3}{|l|}{ Current Gl edema } \\
\hline Positive & $1.96[0.76,5.07]$ & 0.164 \\
\hline \multicolumn{3}{|c|}{ Current laryngeal edema } \\
\hline Positive & $2.044[0.688,6.075]$ & 0.236 \\
\hline \multicolumn{3}{|c|}{ Long-term prophylaxis } \\
\hline Positive & $2.73[0.88,8.41]$ & 0.081 \\
\hline \multicolumn{3}{|l|}{ Disease control } \\
\hline Partly controlled & $2.71[0.82,8.97]$ & 0.102 \\
\hline Not controlled & $10.77[1.78,65.06]$ & 0.010 \\
\hline
\end{tabular}

situation in China represents the current status of many countries with limited treatment options. Considering that the general status of Chinese HAE patients has not been evaluated and that condition-specific questionnaires, including AE-QoL and HAE-QoL, do not have validated translated versions, we chose the SF-36 questionnaire to assess the general quality of life in patients. Throughout the study, we found that the HRQoL scores of Chinese HAE patients were significantly lower than those of the general population in all dimensions, which is in accordance with the results in other countries. The population norm was based on a randomized stratified multistage study that included 3,214 people from five geographically representative cities and provided a reliable estimate of the HRQoL in the Chinese population. The HAE cohort in this article has a similar sex ratio to the SF-36 norm cohort (female percentage: $54.81 \%$ vs $52.21 \%)$. Regarding age composition, the percentage of the 30-39 age group in the HAE cohort was higher $(43.27 \%$ vs $22.90 \%)$ than that in the SF-36 norm cohort, while the percentage of the 60-80 age group was lower (4.81\% vs $16.49 \%)$. Considering that young people tend to score higher on HRQoL scales [25], the fact that HAE patients reported significantly lower HRQoL scores supported the conclusion that HAE has a negative influence on patient quality of life.
Table 4 Logistic regression analysis of HAE patients with an MCS < 50

\begin{tabular}{|c|c|c|}
\hline & Multivariate logistic & esults \\
\hline & OR [95\% Cl] & $\mathrm{p}$ value \\
\hline$\overline{\text { Sex }}$ & & \\
\hline Female & $1.19[0.43,3.33]$ & 0.740 \\
\hline Age & & \\
\hline $31-44$ & $1.82[0.51,6.50]$ & 0.357 \\
\hline$\geq 45$ & $0.64[0.16,2.53]$ & 0.527 \\
\hline Onset age & & \\
\hline $11-20$ & $0.73[0.16,3.32]$ & 0.683 \\
\hline$\geq 21$ & $1.54[0.28,8.40]$ & 0.615 \\
\hline Current skin edema & & \\
\hline Positive & $0.55[0.14,2.10]$ & 0.379 \\
\hline Current Gl edema & & \\
\hline Positive & $1.60[0.54,4.77]$ & 0.396 \\
\hline Current laryngeal ed & & \\
\hline Positive & $4.75[1.09,20.69]$ & 0.038 \\
\hline Long-term prophyla & & \\
\hline Positive & $3.10[0.95,10.12]$ & 0.061 \\
\hline Disease control & & \\
\hline Partly controlled & $2.01[0.62,6.56]$ & 0.245 \\
\hline Not controlled & $1.783 \times 10^{9}(0,+\infty)$ & 0.998 \\
\hline
\end{tabular}

Further subgroup analysis helped us identify patients with decreased quality of life. Women reported lower scores than men in all dimensions except for BP and the PCS. We speculate that estrogen exacerbation may somewhat account for this difference [26]. In addition, women appear to report more adverse reactions to danazol than men [27], which may also contribute to this observation. Patients with mucosal edema have lower RF (i.e., role function) scores than those without. Considering that the main forms of mucosal edema are gastrointestinal edema (usually manifested as severe abdominal pain) and laryngeal edema (manifested as dyspnea or even suffocation), it is reasonable that mucosal edema disturbs daily lives to a large extent. No significant difference was observed between different disease subtypes (type 1 or type 2), probably because of the similar phenotypes in the two forms of C1-INH HAE. The limited number of type 2 patients may have also interfered with the comparison. However, considering that the proportion of type $2 \mathrm{HAE}$ among Chinese patients is lower than that among patients in Europe and America, it was reasonable to include very few type 2 patients in our study. No significant differences were observed between different age groups, which is consistent with the Brazilian study [19]. However, the Swedish study reported a negative correlation between age and quality of life in attack-free periods [28]. 
In terms of disease severity and HRQoL, no significant correlation was found, which is consistent with previous studies $[18,19]$. It may be that because HAE has an extensive influence on the quality of life, even patients who are defined as having mild severity (skin edema less than once per month) would perceive their lives as significantly affected. However, our study found that better disease control was associated with higher SF-36 scores in many dimensions. Physical indicators were more closely related to the disease control state.

We did not observe an association between longterm prophylaxis and HRQoL, which is in accordance with the Swedish study in 2017 [17]. It is thought that patients who do not need long-term prophylaxis usually have much milder disease severity and that their QoL is accordingly less affected by the disease. Among patients who took danazol for long-term prophylaxis, no correlation was found between HRQoL and danazol dosage when all patients were analyzed on the whole. However, considering the side effects of danazol, especially in female patients, it is reasonable to see worse social activity, $\mathrm{RE}$ and $\mathrm{MH}$ status in female HAE patients who took relatively larger dosages of danazol. Admittedly, the relatively small sample size and the nature of this retrospective study may limit further findings.

Multivariate logistic regression was performed to examine whether sex, age, onset age, disease phenotype and disease control degree were possible predictors of decreased physical or mental component summary scores in SF-36. These factors were selected based on clinical experience and prior literature [17]. Uncontrolled disease was a predictor of PCSs lower than 50 (the normal reference), indicating an association between edematous attacks and impaired physical function. Laryngeal edema was a predictor of MCSs lower than 50 (the normal reference), suggesting that the fear of suffocation may reduce the mental quality of life. Nevertheless, studies using larger samples are needed to find effective predictors for low quality of life scores, thereby providing a reference for therapeutic plans.

There are several limitations in the present study. First, this study included patients who were regularly followed up in a national medical center, which means they may be more seriously ill and may have lower quality of life than other patients. This may have led to an overestimation of HRQoL reduction. Second, only 104 patients were included, although this is an acceptable number considering the rarity of HAE and the difficulty of diagnosis in China. Another limitation is that the study population did not include children, who should be investigated in future research.

\section{Conclusion}

In conclusion, Chinese HAE patients reported significantly lower HRQoL scores than the general population. Unsatisfactory disease control is a risk factor for decreased PCSs. Laryngeal edema is a risk factor for decreased MCSs.

\section{Methods}

\section{Study population}

Patients who presented to the Department of Allergy of the Peking Union Medical College Hospital (PUMCH) with a final diagnosis of HAE type 1 or 2 from 1983 to 2017 were identified through medical records. The inclusion criteria were as follows: 1) a history of recurrent angioedema without urticaria, and/or recurrent attacks of abdominal pain and vomiting, and/or laryngeal edema; 2) decreased $\mathrm{C} 1$ inhibitor levels and function confirmed by repeated measurements of type $1 \mathrm{HAE}$; or 3) normal or slightly increased serum $\mathrm{C} 1$ inhibitor levels and lower function confirmed by repeated measurements of type $2 \mathrm{HAE}$. Although some patients described their family members having similar symptoms, only patients with medical records in our center were included in this study. Among the 400 HAE patients identified, 129 were followed regularly in our center. Most of these patients are probands, which means that they usually present with more severe symptoms than their family members. Two patients younger than 18 years old were excluded from the study. A web-based questionnaire and informed consent were sent to the above $127 \mathrm{pa}$ tients, and a total of 104 patients completed the webbased questionnaire independently or with the help of family members.

\section{Questionnaire development}

The questionnaire was developed using the wjx online survey platform (www. Wjx.cn). The questionnaire contains mostly close-ended questions with defined response categories. A few questions asked participants to provide descriptive information in an open-ended text box. A pilot questionnaire was sent to 7 volunteering patients to develop the final version. The final version of the questionnaire was sent to the above HAE patients. Participants were informed about the survey, including the purpose, the agency conducting the research and privacy protection. The survey had five sections measuring demographic data, body characteristics, clinical characteristics, socioeconomic issues and HRQoL (using SF36 for the last).

\section{Sf-36}

SF-36 is a standardized patient-reported survey of generic health measuring eight health domains, including $\mathrm{PF}$, limitations in daily role functioning due to physical 
problems (RP), BP, general health (GH), vitality (VT), SF, limitations in daily role functioning due to emotional problems (RE), and $\mathrm{MH}$, and it also has an item asking respondents about health changes over the past year. Scores for each domain range from 0 to 100, with higher scores indicating a better health state. Scores on each scale were calculated based on the general survey algorithm. The PCS and the MCS were constructed from eight domains representing PF well-being and emotional well-being. The PCS and MCS were calculated by Optum's PRO CoRE Software (Pfizer Inc., version 1.3). A standardized Mandarin version of SF-36 was used in this study.

\section{Clinical severity score}

The clinical severity score was expressed using values from 0 to 10 as previously proposed [29]. The score was calculated by considering the age at the onset of disease $(0-5$ years $=3$ points, $6-10$ years $=2$ points, $11-20$ years $=1$ point, $>20$ years $=0$ points), clinical manifestations $($ skin edema $=1$ point, painful abdominal edema $=$ 2 points, laryngeal edema $=2$ points, other clinical manifestations $=1$ point), and the need for long-term prophylaxis (yes $=1$ point).

\section{Chinese population norm}

The Chinese population norm was based on a study by $\mathrm{He}$ et al. [25]. A random sample $(n=3214)$ of Chinese adults in five cities of mainland China was collected and analyzed.

\section{Disease control status}

The survey asked patients to report their disease control status in a close-ended question. Disease completely controlled was defined as a patient experiencing no edematous attack at present. Disease partly controlled was defined as a patient experiencing decreased severity after diagnosis and treatment. Disease not controlled was defined as a patient experiencing the same or increased severity after diagnosis with or without treatment.

\section{Statistical analysis}

The data were exported via the wjx online survey platform and were checked through internal error detection. All statistical analyses were performed using SPSS 23.9 (SPSS, Inc., USA) software. Descriptive statistics for demographic information were calculated. The results were expressed as either the mean \pm standard deviation [SD] for normally distributed data or the median plus interquartile range $[\mathrm{IQR}]$ for nonnormal data. Categorical data were summarized as the percentage of the total group. Differences in quantitative data distributions between patient subgroups were compared using Student's $t$-test for normally distributed data and by the Wilcoxon rank-sum test or Kruskal-Wallis test for nonnormal data. Differences in frequencies for categorical data were compared using the $\chi^{2}$ test. Spearman correlation analysis was applied to explore the correlation between two variables. Full-model logistic regression analysis was used to determine the value of sex, age, onset age, disease phenotype and disease control degree in predicting decreased PCSs and MCSs. A $p$ value of $<0.05$ was considered statistically significant.

\section{Additional files}

Additional file 1: Table S1. Health-related quality of life in Chinese HAE patients. (DOCX $15 \mathrm{~kb}$ )

Additional file 2: Table S2. Correlation between Clinical Severity Score and HRQOL (Spearman correlation). (DOCX 15 kb)

Additional file 3: Table S3. Correlation between disease control and HRQoL (Spearman correlation). (Disease control status: $1=$ completely controlled, 2 = partly controlled; $3=$ no improvement in disease after diagnosis). (DOCX $15 \mathrm{~kb}$ )

Additional file 4: The dataset suppoting the conclusions of this article. (XLSX $21 \mathrm{~kb})$

\section{Abbreviations}

AE-QoL: angioedema quality of life questionnaire; BP: Bodily pain; C1INH: Complement 1 inhibitor; Cl: Confidence interval; DLQI: Dermatology life quality index; FFP: Fresh frozen plasma; GH: General health; HAE: Hereditary angioedema; HRQoL: Health-related quality of life; IQR: Interquartile range;

MCS: Mental component score; MH: Mental health; PCS: Physical component score; PF: Physical functioning; PRO: Patient-reported outcome;

RE: Limitations in daily role functioning due to emotional problems; RP: Limitations in daily role functioning due to physical problems; SD: Standard deviation; SF: Social functioning; SF-36: Short-form health survey; VT: Vitality

\section{Acknowledgments}

We thank all the patients for their support in this research.

\section{Authors' contributions}

S. Liu, X. Wang and Y. Zhi conceptualized the study. S. Liu, Y. Xu acquired the data. S. Liu, X. Wang, Q. Xu and Y. Zhi interpreted the data. S. Liu and Y. Zhi prepared the manuscript. All authors participated in reviewing and editing the manuscript and in the approval of the finalized manuscript.

\section{Funding}

This work was supported by the National Natural Science Fund (No. 81472870), the CAMS Innovation Fund for Medical Sciences (CIFMS) (No. 2016-12M-1-002), and the National Key Research and Development Program of China (No. 2016YFC0901501).

Availability of data and materials

The datasets supporting the conclusions of this article are included within the article and its Additional file 4: Table S4.

\section{Ethics approval and consent to participate}

The study protocol was approved by the Research and Ethics Board of the PUMCH. Informed consent was obtained from all patients before the study questionnaires were completed.

Consent for publication

All authors have given consent for publication.

Competing interests

The authors have no conflicts of interest to disclose. 


\section{Author details}

'Department of Allergy, Peking Union Medical College Hospital, Chinese Academy of Medical Sciences and Peking Union Medical College, Beijing 100730, China. ${ }^{2}$ School of Clinical Medicine, Chinese Academy of Medical Sciences and Peking Union Medical College, Beijing, China. ${ }^{3}$ Key Laboratory of Endocrinology of National Health Commission, Department of Endocrinology, Peking Union Medical College Hospital, Chinese Academy of Medical Science and Peking Union Medical College, Beijing 100730, China. ${ }^{4}$ Department of Epidemiology and Biostatistics, Institute of Basic Medical Sciences Chinese Academy of Medical Sciences, School of Basic Medicine, Peking Union Medical College, Beijing 100005, China. ${ }^{5}$ Center of Environmental and Health Sciences, Chinese Academy of Medical Sciences, Peking Union Medical College, Beijing 100005, China.

Received: 4 March 2019 Accepted: 18 July 2019

Published online: 08 August 2019

\section{References}

1. Betschel S, Badiou J, Binkley K, Hebert J, Kanani A, Keith P, et al. Canadian hereditary angioedema guideline. Allergy Asthma Clin Immunol. 2014;10(1): 50.

2. Maurer M, Magerl M, Ansotegui I, Aygoren-Pursun E, Betschel S, Bork K, et al. The international WAO/EAACI guideline for the management of hereditary angioedema-the 2017 revision and update. Allergy. 2018;73(8):1575-96.

3. Aygören-Pürsün E, Magerl M, Maetzel A, Maurer M. Epidemiology of bradykinin-mediated angioedema: a systematic investigation of epidemiological studies. Orphanet Journal of Rare Diseases. 2018;13(1):73.

4. Zanichelli A, Longhurst HJ, Maurer M, Bouillet L, Aberer W, Fabien V, et al. Misdiagnosis trends in patients with hereditary angioedema from the realworld clinical setting. Ann Allergy Asthma Immunol. 2016;117(4):394-8.

5. Xu YY, Jiang $Y$, Zhi $Y X$, Yin J, Wang $L L$, Wen $L P$, et al. Clinical features of hereditary angioedema in Chinese patients: new findings and differences from other populations. Eur J Dermatol. 2013;23(4):500-4

6. Caballero T, Prior N. Burden of illness and quality-of-life measures in angioedema conditions. Immunol Allergy Clin N Am. 2017;37(3):597-616.

7. Jindal NL, Harniman E, Prior N, Perez-Fernandez E, Caballero T, Betschel S. Hereditary angioedema: health-related quality of life in Canadian patients as measured by the SF-36. Allergy Asthma Clin Immunol. 2017;13:4.

8. Weller K, Magerl M, Peveling-Oberhag A, Martus P, Staubach P, Maurer M. The angioedema quality of life questionnaire (AE-QoL) - assessment of sensitivity to change and minimal clinically important difference. Allergy. 2016;71(8):1203-9.

9. Lumry WR, Castaldo AJ, Vernon MK, Blaustein MB, Wilson DA, Horn PT. The humanistic burden of hereditary angioedema: impact on health-related quality of life, productivity, and depression. Allergy Asthma Proc. 2010;31(5): 407-14.

10. Xu YY, Zhi YX, Liu RL, Craig T, Zhang HY. Upper airway edema in 43 patients with hereditary angioedema. Ann Allergy Asthma Immunol. 2014;112(6): 539-44 e1.

11. Rubinstein E, Stolz LE, Sheffer AL, Stevens C, Bousvaros A. Abdominal attacks and treatment in hereditary angioedema with C1-inhibitor deficiency. BMC Gastroenterol. 2014;14:71

12. Fouche AS, Saunders EF, Craig T. Depression and anxiety in patients with hereditary angioedema. Ann Allergy Asthma Immunol. 2014;112(4):371-5.

13. Caballero T, Aygoren-Pursun E, Bygum A, Beusterien K, Hautamaki E, Sisic Z, et al. The humanistic burden of hereditary angioedema: results from the burden of illness study in Europe. Allergy Asthma Proc. 2014;35(1):47-53.

14. Bonner N, Abetz-Webb L, Renault L, Caballero T, Longhurst H, Maurer M, et al. Development and content validity testing of a patient-reported outcomes questionnaire for the assessment of hereditary angioedema in observational studies. Health Qual Life Outcomes. 2015:13:92.

15. Poon E, Seed PT, Greaves MW, Kobza-Black A. The extent and nature of disability in different urticarial conditions. Br J Dermatol. 1999;140(4):667-71.

16. Bouillet L, Launay D, Fain O, Boccon-Gibod I, Laurent J, Martin L, et al. Hereditary angioedema with C1 inhibitor deficiency: clinical presentation and quality of life of 193 French patients. Ann Allergy Asthma Immunol. 2013:111(4):290-4.

17. Nordenfelt $P$, Nilsson M. Lindfors a. Bjorkander J. Health-related quality of life in relation to disease activity in adults with hereditary angioedema in Sweden. Allergy Asthma Proc: Wahlgren CF; 2017.
18. Aabom A, Andersen KE, Perez-Fernandez E, Caballero T, Bygum A. Healthrelated quality of life in Danish patients with hereditary angioedema. Acta Derm Venereol. 2015;95(2):225-6.

19. Gomide MA, Toledo E, Valle SO, Campos RA, Franca AT, Gomez NP, et al. Hereditary angioedema: quality of life in Brazilian patients. Clinics (Sao Paulo). 2013;68(1):81-3.

20. Jolles S, Williams P, Carne E, Mian H, Huissoon A, Wong G, et al. A UK national audit of hereditary and acquired angioedema. Clin Exp Immunol. 2014:175(1):59-67.

21. Sanchez MD, Cuervo J, Rave D, Clemen G, Yepes-Nunez JJ, Ortiz-Reyes B, et al. Hereditary angioedema in Medellin (Colombia): clinical evaluation and quality of life appraisal. Biomedica. 2015;35(3):419-28.

22. Bygum A, Andersen KE, Mikkelsen CS. Self-administration of intravenous C1inhibitor therapy for hereditary angioedema and associated quality of life benefits. Eur J Dermatol. 2009:19(2):147-51.

23. Lumry WR, Craig T, Zuraw B, Longhurst H, Baker J, Li HH, et al. HealthRelated Quality of Life with Subcutaneous C1-Inhibitor for Prevention of Attacks of Hereditary Angioedema. J Allergy Clin Immunol Pract. 2018;6(5): 1733-41.e3.

24. Weller K, Maurer M, Fridman M, Supina D, Schranz J, Magerl M. Healthrelated quality of life with hereditary angioedema following prophylaxis with subcutaneous C1-inhibitor with recombinant hyaluronidase. Allergy Asthma Proc. 2017;38(2):143-51.

25. Rui W, Cheng W, Ma XQ, Zhao YF, Yan XY, Jia H. Health-related quality of life in Chinese people: a population-based survey of five cities in China. Scandinavian journal of public health. 2011;39(4):410-8.

26. Walford HH, Zuraw BL. Current update on cellular and molecular mechanisms of hereditary angioedema. Ann Allergy Asthma Immunol. 2014; 112(5):413-8.

27. Bork K, Bygum A, Hardt J. Benefits and risks of danazol in hereditary angioedema: a long-term survey of 118 patients. Ann Allergy Asthma Immunol. 2008;100(2):153-61.

28. Nordenfelt P, Dawson S, Wahlgren CF, Lindfors A, Mallbris L, Bjorkander J. Quantifying the burden of disease and perceived health state in patients with hereditary angioedema in Sweden. Allergy Asthma Proc. 2014;35(2): 185-90

29. Bygum A, Fagerberg CR, Ponard D, Monnier N, Lunardi J, Drouet C. Mutational spectrum and phenotypes in Danish families with hereditary angioedema because of C1 inhibitor deficiency. Allergy. 2011:66(1):76-84.

\section{Publisher's Note}

Springer Nature remains neutral with regard to jurisdictional claims in published maps and institutional affiliations.

Ready to submit your research? Choose BMC and benefit from:

- fast, convenient online submission

- thorough peer review by experienced researchers in your field

- rapid publication on acceptance

- support for research data, including large and complex data types

- gold Open Access which fosters wider collaboration and increased citations

- maximum visibility for your research: over $100 \mathrm{M}$ website views per year

At BMC, research is always in progress.

Learn more biomedcentral.com/submissions 